\title{
Motivación para la práctica físico-deportiva y Síndrome de Burnout en estudiantes de Ciencias de la Salud
}

\author{
Ana Belén Barragán, María del Carmen Pérez-Fuentes, María del Mar Molero, \\ José Jesús Gázquez, África Martos y María del Mar Simón \\ Universidad de Almería (España)
}

\begin{abstract}
Objetivo: Analizar los motivos de la práctica físico-deportiva y el burnout en estudiantes de ciencias de la salud, atendiendo al género. Así como, determinar la relación entre el estado de salud, la edad y el síndrome de burnout. Método: La muestra está compuesta por un total de 188 estudiantes universitarios pertenecientes a diferentes titulaciones de Ciencias de la Salud con edades comprendidas entre los 18 y 54 años Para llevar a cabo el estudio se implementó el Cuestionario de contenido de los objetivos en el ejercicio y el Maslach Burnout Inventory. Y para el análisis de datos se utilizó el programa estadístico SPSS 23. Resultados: En cuanto al síndrome de burnout según el género, encontramos que en el factor agotamiento, el grupo de las mujeres obtienen puntuaciones mayores, aunque estas diferencias no son estadísticamente significativas, respecto al grupo de los hombres. Respecto a lo motivos de la práctica físico-deportiva en función del género, aunque no existen diferencias significativas, el grupo de las mujeres obtiene puntuaciones más altas en el factor imagen, desarrollo de habilidad, afiliación social, y en el reconocimiento social, que el grupo de los hombres. Sin embargo, el grupo de los hombres solamente obtiene puntuaciones más altas en el factor dirección de la salud. Conclusiones: En esta investigación hallamos que no existen diferencias estadísticamente significativas en las puntuaciones de los cinco factores de la escala GCEQ, y tampoco en los tres factores, agotamiento, cinismo y eficacia profesional según el género en los estudiantes universitarios de Ciencias de la Salud.
\end{abstract}

Palabras clave: Ejercicio, motivación, burnout, deportes, estudiantes del Área de la Salud.

Motivation for physical-sports practice and burnout syndrome in students of Health Sciences. Objective: To analyze the motives of the physical-sport practice and the burnout in students of Health Science, attending to the gender. As well as, determine the relationship between health status, age and burnout syndrome. Method: The sample is composed of 188 University students belonging to different degrees in Health Science with ages between 18 and 54 years. To carry out the study, the questionnaire of content of the objectives in the exercise was implemented and the Maslach Burnout Inventory. The statistical program SPSS 23 was used for the data analysis. Results: Regarding burnout syndrome according to gender, we found that in the depletion factor, the group of women obtained higher scores, although these differences are not statistically significant, with respect to the group of men. Regarding the reasons for the physical-sporting practice according to gender, although there are no significant differences, the group of women obtains higher scores in the image factor, skill development, social affiliation, and in social recognition, than the group of men. However, the group of men only scores higher in the health direction factor. Conclusions: In this research we find that there are no statistically significant differences in the scores of the five factors of the GCEQ scale, nor in the three factors, exhaustion, cynicism and professional efficacy according to gender in the university students of Health Science.

Keywords: Exercise, motivation, burnout, sports, students of the Health Area.

Correspondencia: $\mathrm{M}^{\mathrm{a}}$ del Carmen Pérez-Fuentes. Departamento de Psicología. Universidad de Almería. Ctra. de Sacramento, s/n. C.P.: 04120. Almería (España).E-mail: mpf421@ual.es 
En los últimos años, se produce un aumento en la práctica deportiva por parte de la sociedad en general. Concretamente, en España se está generalizando el interés hacia esta (Moreno-Murcia, Marcos-Pardo, y Huéscar, 2016). Cuando hablamos de la práctica físico-deportiva, hacemos referencia a la actividad de ocio que contribuye con mayores beneficios físicos, sociales y psicológicos (Úbeda-Colomer, Molina, y Campos, 2016). Son numerosos los estudios y las encuestas que revelan datos sobre los múltiples beneficios que aporta a la salud, tanto en jóvenes como adultos (Pérez-Muñoz y Sánchez-Vegas, 2015; Zumeta, Basade, Telletxea, Amutio, y Bobowik, 2016).

En este sentido, la mayoría de los estudios realizados, en todo el mundo, coinciden en que las mujeres realizan menos actividad física que los hombres y señalan que su estado de salud es peor (Castillo y Giménez, 2011). Así, en el estudio realizado por Díaz, Santana, Rodríguez, y Moreno (2014) se obtuvieron diferencias en los porcentajes entre hombres y mujeres que realizan actividad física, destacando el grupo de las mujeres con menor porcentaje. No obstante, no solamente disminuye la práctica físico-deportiva en función del género, sino también dependiendo de la edad, en especial en la etapa adolescente (Crane y Temple, 2015). Cabe señalar que en la población adolescente la práctica deportiva se ha visto mermada a causa del sedentarismo, y más en concreto entre las jóvenes. Según la Encuesta de Hábitos Deportivos en España de 2015, en el último año la mitad de los sujetos con 15 años realizó deporte de forma periódica o esporádica. Además, la edad y el género han sido variables determinantes, ya que la población más joven ha obtenido porcentajes más elevados respecto a los adultos de 75 años en adelante. Y, en función del género, también existen diferencias en la práctica deportiva siendo los porcentajes más altos en el grupo de los hombres que en el de las mujeres (Ministerio de Educación, Cultura y Deporte, 2015).

Por otro lado, en el desarrollo de la práctica físico-deportiva influyen una serie de factores psicológicos y psicosociales, entre ellos la motivación (Jiménez-Torres, Godoy-Izquierdo, y Godoy-García, 2012). Los procesos motivacionales dirigen la conducta del sujeto hacia la práctica físico-deportiva y hacen que permanezca con el tiempo la decisión que fue tomada, debido al interés que origina la participación en una actividad. En este sentido, existen diferentes razones por las que los individuos se implican en la práctica físico-deportiva. Si atendemos a los jóvenes y/o universitarios encontramos motivos como: mejorar la forma física, la diversión, deseo de logro, competición, relaciones con otros iguales, mejorar la salud, etc. En cuanto a las personas adultas, los motivos por los que realizan estas actividades irían más en consonancia con el factor social (Moreno, Águila, y Borges, 2011). Igualmente, existen diferencias entre hombres y mujeres en cuanto a los motivos por los que realizan actividades deportivas. En el caso del grupo de las mujeres, la apariencia, el control del peso, además, de la relación social, mientras que, los hombres, persiguen una mejora en la forma física, la competición y el rendimiento (Fernández, Sáenz-López, Almagro, y Conde, 2015). 
Además, otro motivo para realizar la práctica físico-deportiva, sería el placer o la alegría (Jiménez-Torres, Godoy-Izquierdo, y Godoy-García, 2012). Igualmente, otro de los motivos por los que la población realiza actividad deportiva según la Encuesta de Hábitos Deportivos en España 2015 es la salud y considerarla como una forma de relajación (Ministerio de Educación, Cultura y Deporte, 2015).

Como podemos observar la percepción de la utilidad del deporte atendiendo al género es distinta, por tanto, es importante satisfacer la necesidad de competencia, autonomía, relaciones sociales para valorar la práctica deportiva como algo ventajoso (Amado, Sánchez-Miguel, Leo, Sánchez-Oliva, y García-Calvo, 2014).

La práctica deportiva no es solamente beneficiosa para aspectos físicos, sino también para prevenir estados psicológicos y las patologías por las que el individuo puede verse afectado, como puede ser la depresión, ansiedad (Sampedro, 2015), irritabilidad, etc. Diferentes investigaciones han demostrado las ventajas de ser una persona físicamente activa tanto a nivel físico como psicológico, por ejemplo, en relación al síndrome de burnout el cual se puede ver reducido gracias a los cambios de estilos de vida más saludables (Gonçalves et al., 2015). En este sentido, cuando damos respuesta a una situación de estrés laboral crónico que se prolonga en el tiempo, y los trabajadores presentan altos porcentajes de desmotivación, estamos haciendo referencia a lo que conocemos como el síndrome de burnout (Casa, Rincón, y Vila, 2012).

La prevalencia del síndrome de burnout va en aumento y para desarrollarse tienen que interaccionar factores diversos como la presión laboral y el estrés individual (Barragán et al., 2015). Por tanto, el burnout lo puede desarrollar cualquier individuo que desempeñe una actividad laboral percibida hasta cierto punto como exigente (González, Souto, Freire, Fernández, y González, 2016). Por ejemplo, los cuidadores de personas mayores se ven afectados por una serie de variables que, asociadas entre sí, dan lugar al síndrome de burnout, lo cual perjudica su bienestar y calidad de vida (Gázquez, Pérez-Fuentes, Molero, y Mercader, 2015; Pérez-Fuentes et al., 2014). Por otro lado, en el estudio llevado a cabo por Casa, Rincón, y Vila (2012), se halló que la prevalencia del burnout en la muestra de profesionales de enfermería del servicio de urgencias y el de una residencia de adultos fue de un nivel medio. En el primer grupo, se encontraron niveles medios-altos frente a los enfermeros de la residencia, que presentaban niveles bajos.

Hasta ahora, solamente se había tratado el síndrome de burnout en el ámbito sanitario, pero este síndrome se extiende a todos los ámbitos, entre ellos el educativo. El ámbito académico es uno de los más vulnerables respecto a la aparición del burnout, debido a diferentes factores de interacción, organización, personales, etc. (RodríguezMantilla y Fernández-Díaz, 2017). La investigación sobre el burnout se han centrado principalmente en el profesorado (Rodríguez, Sola, y Fernández, 2017). Más adelante, se ha extendido a los estudiantes, denominándolo como burnout académico. En este 
sentido, la universidad desempeña un rol importante en la formación de los alumnos (Esteban, Bernardo, Tuero, Cerezo, y Núñez, 2016). Las nuevas reformas educativas y lo que conllevan, pueden incrementar la presión en el alumnado. En el ámbito universitario, el burnout ha afectado especialmente a las titulaciones, que por sus características, presentan una alta carga académica y elevados niveles de estrés (Bolaños y Rodríguez, 2016). En algunas ocasiones, la permanencia en la universidad se ve influenciada por este fenómeno y otras variables influyentes (Esteban, Bernardo, Tuero, Cervero, y Casanova, 2017) que pueden conllevar al abandono.

En diferentes estudios se ha encontrado una prevalencia de burnout en estudiantes universitarios, tanto en agotamiento físico y/o emocional, cinismo y falta de eficacia profesional. De igual forma, se indica que los estudiantes de enfermería tienen una alta propensión a sufrir este síndrome, puesto que el personal de enfermería en su puesto de trabajo tiene una alta probabilidad de padecerlo (Vizoso y Arias, 2016).

El burnout también se ve afectado y depende de las variables sociodemográficas, en cuanto al género encontramos que en el estudio llevado a cabo por Rosales (2012) se halló que la mayor parte de los estudiantes encuestados manifestaron el síndrome de burnout leve, y el género femenino fue el más afectado. En cambio, en la investigación llevada a cabo por Peralta-Ayala y Moya (2017) señalaron que no existen diferencias significativas entre el grupo de hombres y mujeres en cuanto al síndrome de burnout. Respecto a la variable edad, en este mismo estudio si existieron diferencias significativas entre el grupo de edad de 25 a 30 años y el de 20 a 24 años, donde el primer grupo mostro elevados niveles de cansancio emocional y el segundo grupo niveles medios, en despersonalización los dos grupos presentan niveles bajos, y en eficacia profesional ambos muestran niveles medios.

Atendiendo a los resultados de otras investigaciones, este estudio tiene como objetivo analizar los motivos de la práctica físico-deportiva y el burnout en estudiantes de ciencias de la salud, atendiendo al género. Así como, determinar la relación entre el estado de salud, la edad y el síndrome de burnout.

\section{MÉTODO}

\section{Participantes}

La muestra está compuesta por un total de 188 estudiantes universitarios pertenecientes a diferentes titulaciones de Ciencias de la Salud con edades comprendidas entre los 18 y 54 años $(M=32.05 ; D T=9.44)$. Del total de participantes, 47 son hombres $(25 \%)$ y 141 son mujeres $(75 \%)$. Respecto al estado civil de la muestra el $56.4 \%$ $(N=106)$ era soltero/a, el $3.7 \%(N=7)$ divorciado/a o separado/a, el 39,4\% $(N=74)$ era casado/a o pareja estable, y por último, el $0.5 \%(N=1)$ viudo/a. 


\section{Instrumentos}

Cuestionario ad hoc, con el objetivo de conocer las características sociodemográficas de los participantes, además se recogen datos sobre la salud percibida.

Goal Content for Exercise Questionnaire (GCEQ; Sebire et al., 2008), concretamente la adaptación española de Moreno-Murcia, Marcos-Pardo, y Huéscar (2016). Cuestionario de contenido de los objetivos en el ejercicio que mide las razones por las que las personas realizan ejercicio físico. Este cuestionario está compuesto por un total de 20 ítems agrupados en cinco factores que son: afiliación social, imagen, dirección de la salud, reconocimiento social y desarrollo de habilidades, cada uno de estos factores consta de 4 ítems. La modalidad de respuesta se basa en una escala Likert de 7 puntos, donde se le pide al sujeto que indique en qué medida los objetivos planteados son importantes para él en el ejercicio físico, siendo 1 nada importante y 7 extremadamente importante.

Maslach Burnout Inventory (MBI; Maslach y Jackson, 1986), concretamente, se utilizó la adaptación para estudiantes (Schaufeli, Martínez, Pinto, y Salanova, 2002). Este cuestionario evalúa los sentimientos y actitudes los estudiantes hacia su actividad académica, midiendo el desgaste a través de la frecuencia y la intensidad de este. Para ello, se vale de 11 ítems, agrupados en tres subescalas (agotamiento emocional, despersonalización y eficacia académica). Así, el primer factor agotamiento emocional, valora la vivencia o sentimiento de encontrarse física, mental y emocionalmente exhausto, y con una sensación de no poder dar más de sí mismo frente a las actividades académicas. La subescalas de despersonalización o incredulidad, evalúa sus estudios, evidenciada por la autocrítica, desvalorización, pérdida del interés y de la trascendencia y valor frente al estudio. Por último, la subescala "realización profesional", evalúa los sentimientos de autoeficacia y realización personal en los estudios. Cada ítem es valorado a través de una escala tipo Likert de 7 puntos (siendo 0 nunca y 6 todos los días) a través de los cuales se pide al sujeto que valore la frecuencia con la que siente lo indicado en cada enunciado.

\section{Procedimiento y análisis de datos}

La participación fue voluntaria y todos los sujetos que realizaron el cuestionario fueron informados de los objetivos del estudio y el modo de cumplimentar éste. Además, se les informo que sus respuestas iban a ser totalmente anónimas y el uso de los datos confidencial. El cuestionario fue cumplimentado de forma individual. Para el análisis de datos se utilizó el programa estadístico SPSS. 23. Los parámetros descriptivos se llevaron a cabo mediante un análisis de frecuencias. Igualmente, para conocer si existían diferencias significativas en la práctica físico-deportiva y en la salud percibida, en función del género, se utilizó la prueba $t$ de Student. Y por último, para 
analizar la relación entre la salud percibida, la edad, ejercicio y burnout se empleó la correlación de Pearson.

\section{RESULTADOS}

Motivos para la realización de la actividad física en estudiantes de ciencias de la salud

En cuanto a los porcentajes obtenidos sobre el estado de salud de los estudiantes universitarios de Ciencias de la Salud según el género, donde el $85.7 \%$ de las mujeres señala tener un estado de salud regular respecto al $14.3 \%$ de los hombres. Así mismo, el $68.1 \%$ de las mujeres destacó tener "muy buena" salud en proporción al $31.9 \%$ de los hombres. No obstante, cabe destacar, que de los que señalaron la opción "muy mala" el 100\% eran hombres.

Por otro lado, se presentan los resultados de las medias obtenidas en el género, al compararlo con las diferentes escalas del GCEQ. En la tabla 1 puede observarse, que aunque no existen diferencias significativas, el grupo de las mujeres obtiene puntuaciones más altas en el factor imagen $\left(t_{(187)}=-.91 ; p=.36\right)$, desarrollo de habilidad $\left(t_{(187)}=-1.15 ; p=.24\right)$, afiliación social $\left(t_{(187)}=-.50 ; p=.61\right)$, y en el reconocimiento social $\left(t_{(187)}=-.26 ; p=.79\right)$ que el grupo de los hombres. Sin embargo, el grupo de los hombres solamente obtiene puntuaciones más altas en el factor dirección de la salud $\left(t_{(187)}=-.14\right.$; $p=.88)$.

Tabla 1. Contenido de los objetivos en el ejercicio según el género. Descriptivos y prueba $t$ de Student

\begin{tabular}{|c|c|c|c|c|c|c|c|c|}
\hline \multirow{3}{*}{ Escalas GCEQ } & \multicolumn{6}{|c|}{ Género } & \multirow{3}{*}{$t$} & \multirow{3}{*}{$p$} \\
\hline & \multicolumn{3}{|c|}{ Hombre } & \multicolumn{3}{|c|}{ Mujer } & & \\
\hline & $N$ & Media & $D T$ & $N$ & Media & $D T$ & & \\
\hline Afiliación social & 47 & 15.43 & 3.65 & 141 & 15.74 & 3.51 & -.50 & .61 \\
\hline Imagen & 47 & 16.48 & 3.78 & 141 & 17.05 & 3.68 & -.91 & .36 \\
\hline Dirección de la salud & 47 & 16.33 & 3.82 & 141 & 16.24 & 3.50 & -.14 & .88 \\
\hline Reconocimiento social & 47 & 15.16 & 3.19 & 141 & 15.30 & 3.18 & -.26 & .79 \\
\hline Desarrollo de habilidad & 47 & 18.52 & 3.88 & 141 & 19.31 & 4.07 & -1.15 & .24 \\
\hline
\end{tabular}

En relación, a la salud percibida según el género de los estudiantes (ver tabla 2) encontramos que el grupo de los hombres $(M=3.35 ; D T=.84)$ obtiene puntuaciones mayores $\left(t_{(187)}=.82 ; p=.41\right)$, aunque estas diferencias no son significativas, respecto al grupo de las mujeres $(M=3.25 ; D T=.67)$.

Tabla 2. Salud percibida según género. Descriptivos y prueba $t$ de Student

\begin{tabular}{|c|c|c|c|c|c|c|c|c|}
\hline & \multicolumn{6}{|c|}{ Género } & \multirow{3}{*}{ 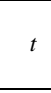 } & \multirow{3}{*}{$p$} \\
\hline & \multicolumn{3}{|c|}{ Hombre } & \multicolumn{3}{|c|}{ Mujer } & & \\
\hline & $N$ & Media & $D T$ & $N$ & Media & $D T$ & & \\
\hline ¿Cómo diría que es su estado de salud? & 46 & 3.35 & .84 & 134 & 3.25 & .67 & .82 & .41 \\
\hline
\end{tabular}


En la tabla 3, se presentan los resultados de la correlación de Pearson entre la edad, salud y los factores de la escala GCEQ, que muestra la existencia de una correlación positiva entre la edad y la afiliación social $(r=.214 ; p<0.01)$, la edad y la imagen ( $r=.173 ; p<0.05)$, la edad y la dirección de la salud $(r=.146 ; p<0.05)$, la edad y el reconocimiento social $(r=.246 ; p<0.01)$, y por último, la edad y el desarrollo de habilidades $(r=.233 ; p<0.01)$. Es decir, le dan mayor importancia los estudiantes universitarios de Ciencias de la Salud al reconocimiento social y al desarrollo de habilidades como objetivo en la práctica físico-deportiva.

Tabla 3. Edad, salud y factores de la escala GCEQ. Correlación de Pearson

\begin{tabular}{|c|c|c|c|c|c|c|c|}
\hline & & $\begin{array}{l}\text { Percepción } \\
\text { de la salud }\end{array}$ & $\begin{array}{c}\text { Afiliación } \\
\text { social }\end{array}$ & Imagen & $\begin{array}{c}\text { Dirección } \\
\text { de la salud }\end{array}$ & $\begin{array}{c}\text { Reconocimiento } \\
\text { social }\end{array}$ & $\begin{array}{c}\text { Desarrollo de } \\
\text { habilidades }\end{array}$ \\
\hline \multirow{3}{*}{ Edad } & Pearson & .074 & $.214 * *$ & $.173 *$ & $.146^{*}$ & $.246^{* *}$ & $.233 * *$ \\
\hline & Sig. & .326 & .003 & .018 & .045 & .001 & .001 \\
\hline & $N$ & 180 & 188 & 188 & 188 & 188 & 188 \\
\hline \multirow{3}{*}{$\begin{array}{l}\text { Percepción de la } \\
\text { salud }\end{array}$} & Pearson & & $.233 * *$ & .129 & .088 & $.162 *$ & $.252 * *$ \\
\hline & Sig. & & .002 & .084 & .239 & .030 & .001 \\
\hline & $N$ & & 180 & 180 & 180 & 180 & 180 \\
\hline \multirow{3}{*}{ Afiliación social } & Pearson & & & .769 *** & $.754 * * *$ & $.793 * * *$ & $.814 * * *$ \\
\hline & Sig. & & & .000 & .000 & .000 & .000 \\
\hline & $N$ & & & 188 & 188 & 188 & 188 \\
\hline \multirow{3}{*}{ Imagen } & Pearson & & & & $.835^{\text {**** }}$ & $.752 * * *$ & $.827 * * *$ \\
\hline & Sig. & & & & .000 & .000 & .000 \\
\hline & $N$ & & & & 188 & 188 & 188 \\
\hline \multirow{3}{*}{$\begin{array}{l}\text { Dirección de la } \\
\text { salud }\end{array}$} & Pearson & & & & & $.866^{* * * *}$ & $.759 * * *$ \\
\hline & Sig. & & & & & .000 & .000 \\
\hline & $N$ & & & & & 188 & 188 \\
\hline \multirow{3}{*}{$\begin{array}{l}\text { Reconocimiento } \\
\text { social }\end{array}$} & Pearson & & & & & & $.782 * * *$ \\
\hline & Sig. & & & & & & .000 \\
\hline & $N$ & & & & & & 188 \\
\hline
\end{tabular}

Síndrome de burnout en estudiantes de ciencias de la salud

En relación a los resultados obtenidos en la correlación de Pearson entre la edad, salud y factores de la escala MBI (ver tabla 4), podemos observar que existe una correlación positiva y significativa entre la edad y el agotamiento $(r=.255 ; p<0.001)$, la edad y la eficacia profesional $(r=.188 ; p<0.05)$. Es decir, las personas con mayor edad suelen presentar un mayor agotamiento y una mayor eficacia profesional. Así mismo, existe una correlación positiva entre el estado de salud y los tres factores de la escala MBI, con el agotamiento $(r=.278 ; p<0.001)$, el cinismo $(r=.221 ; p<0.01)$, y la eficacia profesional $(r=.256 ; p<0.01)$. 
Tabla 4. Edad, salud y factores de la escala MBI. Correlación de Pearson

\begin{tabular}{|c|c|c|c|c|c|}
\hline & & Cinismo & Eficacia profesional & Edad & Percepción de la salud \\
\hline \multirow{3}{*}{ Agotamiento } & Pearson & $.723 * * *$ & .834 **** & $.255^{* * *}$ & $.278 * * *$ \\
\hline & Sig. & .000 & .000 & .000 & .000 \\
\hline & $N$ & 188 & 188 & 188 & 180 \\
\hline \multirow{3}{*}{ Cinismo } & Pearson & & .829 **** & .139 & $.221 * *$ \\
\hline & Sig. & & .000 & .056 & .003 \\
\hline & $N$ & & 188 & 188 & 180 \\
\hline \multirow{3}{*}{ Eficacia profesional } & Pearson & & & $.188^{*}$ & $.256^{* *}$ \\
\hline & Sig. & & & .010 & .001 \\
\hline & $N$ & & & 188 & 180 \\
\hline \multirow{3}{*}{ Edad } & Pearson & & & & .074 \\
\hline & Sig. & & & & .326 \\
\hline & $N$ & & & & 180 \\
\hline
\end{tabular}

$* p<0.05 ; * * p<0.01 ; * * * p<0.001$

En cuanto al síndrome de burnout según el género (ver tabla 5), encontramos que en el factor agotamiento, el grupo de las mujeres $(M=4.11 ; D T=1.53)$ obtienen puntuaciones mayores, aunque estas diferencias no son estadísticamente significativas $\left(t_{(187)}=-1.187 ; p=.237\right)$, respecto al grupo de los hombres $(M=3.81 ; D T=1.40)$. Al igual ocurre con el factor del cinismo, donde las mujeres $(M=3.77 ; D T=1.14)$, alcanzan puntuaciones elevadas $\left(t_{(187)}=-.967 ; p=.335\right)$, en relación al grupo de los hombres $(M=3.58 ; D T=1.27)$. Por último, en el factor eficacia profesional, sigue siendo el grupo de las mujeres $(M=3,92 ; D T=1,22)$, el que consigue puntuaciones más elevadas $\left(t_{(187)}=-.986 ; p=.325\right)$, respecto a las obtenidas por el grupo de los hombres $(M=3.72$; $D T=1.23$ ), pero estas puntuaciones no son estadísticamente significativas.

Tabla 5. Síndrome de Burnout según género. Descriptivos y prueba $t$ de Student

\begin{tabular}{lllllllll}
\hline & \multicolumn{6}{c}{ Género } & \multirow{4}{*}{ Mujer } & \multirow{2}{*}{$t$} \\
\cline { 2 - 7 } & $N$ & Media & $D T$ & $N$ & Media & $D T$ & & \\
\hline Agotamiento & 47 & 3.81 & 1.40 & 141 & 4.11 & 1.53 & -1.187 & .237 \\
\hline Cinismo & 47 & 3.58 & 1.27 & 141 & 3.77 & 1.14 & -.967 & .335 \\
\hline Eficacia profesional & 47 & 3.72 & 1.23 & 141 & 3.92 & 1.22 & -.986 & .325 \\
\hline
\end{tabular}

\section{DISCUSIÓN Y CONCLUSIONES}

Existen diferentes razones por las que las personas se involucran en actividades físico-deportivas que bien pueden responder a la búsqueda de beneficios físicos, psicológicos y sociales (Pérez-Muñoz y Sánchez-Vegas, 2015). Según los resultados obtenidos, podemos observar que el estado de salud de los estudiantes de Ciencias de la Salud según el género, muestran que el grupo de las mujeres obtiene porcentajes más elevados en todos los estados (mala, regular, buena y muy buena), menos en el estado muy mala salud. 
En relación, a los objetivos por los que realizar práctica físico-deportiva según el género obtenemos resultados contradictorios a los encontrados en otros estudios. Así, en esta investigación hallamos que no existen diferencias estadísticamente significativas en las puntuaciones de los cinco factores de la escala GCEQ según el género en los estudiantes universitarios de Ciencias de la Salud. En cambio, otras investigaciones señalan que existen diferencias entre hombres y mujeres en función de los motivos por los que realizar la práctica físico-deportiva (Fernández, Sáenz-López, Almagro, y Conde, 2015). En concreto en el estudio de Díaz, Santana, Rodríguez, y Moreno (2014) encontraron porcentajes más bajos en el grupo de las mujeres respecto al de los hombres. En este sentido, podemos señalar que, en nuestro estudio, al tratarse de una población específica como son los estudiantes de Ciencias de la Salud, no se han encontrado diferencias, ya que están más concienciados sobre los beneficios de la práctica deportiva, y por tanto, es más difícil descubrir diferencias entre ambos grupos. Quizás con otro tipo de muestra de población universitaria o con población general si encontraríamos diferencias según el género.

Igualmente, en el estado de salud no se dan diferencias estadísticamente significativas entre hombres y mujeres, sin embargo en el estudio de Castillo y Giménez (2011) señalaron que el grupo de las mujeres percibe peor la salud general respecto al grupo de los hombres.

Respecto a los resultados de la correlación de Pearson entre la edad y los factores de la escala GCEQ, encontramos que existe una relación positiva y significativa entre la edad y los cinco motivos por los que realizar práctica físico-deportiva, es decir, los estudiantes universitarios de Ciencias de la Salud con mayor edad le dan más importancia al motivo del reconocimiento social y al desarrollo de habilidades. Estos resultados están en línea con los de la Encuesta de Hábitos Deportivos en España de 2015, donde los jóvenes le dan más importancia a la realización de la práctica deportiva por las relaciones sociales y aspecto físico (Ministerio de Educación, Cultura y Deporte, 2015).

Por otro lado, en cuanto a los resultados de la correlación de Pearson entre la edad, salud y factores del MBI, encontramos que existe una relación positiva entre la edad y el agotamiento, la edad y la eficacia profesional, es decir los estudiantes con mayor edad son los que presentan un mayor agotamiento, pero también una mayor eficacia profesional. Lo cual concuerda con el estudio de Peralta-Ayala y Moya (2017) donde el grupo de edad de 25 a 30 años presentó niveles altos de cansancio emocional y el grupo de 20 a 24 años niveles medios.

En relación a la variable género, no se encontraron relaciones estadísticamente significativas entre los tres factores, agotamiento, cinismo y eficacia profesional. Aunque las puntuaciones obtenidas por el grupo de las mujeres en los tres factores fueron mayores respecto al grupo de los hombres. Estos resultados concuerdan con los 
hallados por Peralta-Ayala y Moya (2017) donde tampoco encontraron diferencias estadísticamente significativas en la variable género respecto al síndrome de burnout. En cambio, en el estudio realizado por Rosales (2012) se mostró que la mayor parte de los estudiantes manifestaron un nivel leve del síndrome de burnout y el grupo de las mujeres fue el más afectado por éste. La existencia de diferencias de género en estos estudios puede deberse al tipo de muestra e instrumento empleado. En nuestro caso, si empleáramos otro tipo de muestra y/o instrumento a lo mejor encontraríamos diferencias según el género en los factores del síndrome de burnout.

En definitiva, con la realización de este estudio se puede comprobar como la presencia del burnout, no solamente está presente en el ámbito sanitario en los profesionales de enfermería (Casa, Rincón, y Vila, 2012), sino también está aumentando en el ámbito académico, y al igual que las personas mayores y sus cuidadores se ven afectados por este (Gázquez et al., 2015), los alumnos y el profesorado cada vez más lo están sufriendo y/o manifestando los signos físicos y conductuales que conlleva (Rodríguez, Sola, y Fernández, 2017).

Por tanto, es fundamental prestar atención a los factores que están relacionados o son predisponentes a la aparición del síndrome de burnout en la población universitaria, para tenerlos en cuenta a la hora de intervenir o utilizarlos como herramientas para el afrontamiento de los diferentes signos de ansiedad, estrés, agotamiento, etc., que pueden presentar los estudiantes. Por último, para futuras investigaciones sería interesante aumentar el tamaño de la muestra, realizar comparaciones entre varios grupos de edad y compararlos con estudiantes de otras titulaciones que no estén relacionadas con el ámbito sanitario, para comprobar si la no existencia de diferencias entre hombres y mujeres, es debida al tipo de población encuestada. Por otro lado, la identificación de los motivos por los que los estudiantes universitarios realizan deporte es de gran utilidad, a la hora de desarrollar actividades o programas de intervención, para poder incentivar la práctica de este y aplicarlo al resto de alumnado universitario. Así, se hace necesario fomentar la diversión, el placer y la alegría como motivos a la hora de realizar deporte.

\section{REFERENCIAS}

Amado, D., Sánchez-Miguel, P.A., Leo, F.M., Sánchez-Oliva, D., y García-Calvo, T. (2014). Diferencias de género en la motivación y percepción de utilidad del deporte escolar. Revista Internacional de Medicina y Ciencias de la Actividad Física y el Deporte, 1(56), 651-664.

Barragán, A.B., Martos, A., Cardila, F., Molero, M.M., Pérez-Fuentes, M.C., y Gázquez, J.J. (2015). Variables relacionadas y prevalencia del burnout en profesionales sanitarios. European Journal of Health Research, 1(1), 5-14. 
Bolaños, N., y Rodríguez, N. (2016). Prevalencia del Síndrome de Burnout académico en el estudiantado de Enfermería de la Universidad de Costa Rica. Revista Electrónica Enfermería Actual en Costa Rica, 31, 1-19.

Casa, P.P., Rincón, Y.Z., y Vila, R. (2012). Síndrome de burnout: enfermedad silenciosa. Enfermería Integral: Revista científica del Colegio Oficial de A.T.S de Valencia, 100, 19-24.

Castillo, E., y Giménez, F.J. (2011). Hábitos de práctica de actividad física del alumnado de la universidad de Huelva. Revista Internacional de Medicina y Ciencias de la Actividad Física y el Deporte, 10 (41), 127-144.

Crane, J., y Temple, V. (2015). A systematic review of dropout from organized sport among children and youth. European Physical Education Review, 21(1), 114-131.

Díaz, M.E., Santana, D., Rodríguez, L., y Moreno, V. (2014). Actividad física y estado nutricional en adolescentes. Una visión de género. Antropo, 31, 39-49.

Esteban, M., Bernardo, A., Tuero, E., Cervero, A., y Casanova, J. (2017). Variables influyentes en progreso académico y permanencia en la universidad. European Journal of Education and Psychology, 10(2), 75-81.

Esteban, M., Bernardo, A.B., Tuero, E., Cerezo, R., y Núñez, J.C. (2016). El contexto sí importa: identificación de relaciones entre el abandono de titulación y variables contextuales. European Journal of Education and Psychology, 9(2), 79-88.

Fernández, E.J., Sáenz-López, P., Almagro, B.J., y Conde, C. (2015). Motivos de práctica de actividades física en universitarios activos. EmásF: Revista Digital de Educación Física, $34,52-60$.

Gázquez, J.J., Pérez-Fuentes, M.C., Molero, M.M., y Mercader, I. (2015). Inteligencia emocional y calidad de vida del cuidador familiar de pacientes con demencia. Revista de Comunicación y Salud, 5, 1-15.

Gonçalves, E., Cascaes, F., Graziele, G., Soleman, S.S., Manoel, V., y Silva, R. (2015). Revisión sistemática sobre síndrome de burnout y actividad física en profesores. Educación Física y Deporte, 34 (2), 309-330.

González, R., Souto, A.J., Freire, C., Fernández, R., y González, L. (2016). La autoestima como variable protectora del burnout en estudiantes de fisioterapia. Estudios sobre Educación, 30, 95-113.

Jiménez-Torres, M.G., Godoy-Izquierdo, D., y Godoy, J.F. (2012). Relación entre los motivos para la práctica físico-deportiva y las experiencias de flujo en jóvenes: diferencias en función del sexo. Universitas Psychologica, 11(3), 909-920.

Ministerio de Educación, Cultura y Deporte (2015). Encuesta de Hábitos Deportivos en España 2015. Madrid: Secretaría General Técnica.

Moreno, J.A., Águila, C., y Borges, F. (2011). La socialización en la práctica físico-deportiva de carácter recreativo: predictores de los motivos sociales. Apunts. de Educación Física y Deportes, 103(1), 76-82.

Moreno-Murcia, J.A., Marcos-Pardo, P.J., y Huéscar, E. (2016). Motivos de Práctica FísicoDeportiva en Mujeres: Diferencias entre Practicantes y no Practicantes. Revista de Psicología del Deporte, 25(1), 35-41.

Peralta-Ayala, R., y Moya, T.J. (2017). Síndrome de burnout en estudiantes de odontología. Dominio de las Ciencias, 3(1), 432-442.

Pérez-Muñoz, S., y Sánchez-Vegas, M. (2015). Motivos de participación y satisfacción en actividades cooperativas y no competitivas en el medio natural. Ágora para la Educación Física y el Deporte, 17(3), 284-299. 
Rodríguez, A.M., Sola, T., y Fernández, M. (2017). Impacto del Burnout en el desarrollo profesional del profesorado universitario. Una revisión de la investigación. Revista Electrónica Interuniversitaria de Formación del Profesorado, 20(3), 161-178.

Rodríguez-Mantilla, J.M., y Fernández-Díaz, M.J. (2017). The effect of interpersonal relationships on burnout síndrome in Secondary Education teachers. Psicothema, 29(3), 370-377.

Rosales, Y. (2012). Estudio unidimensional del síndrome de burnout en estudiantes de medicina de Holguín. Revista de la Asociación Española de Neuropsiquiatría, 32(116), 795-803.

Sampedro, P. (2015). Efecto del ejercicio aeróbico y la estimulación ambiental sobre la reducción de los niveles de ansiedad en el envejecimiento. European Journal of Investigation in Health, Psychology and Education, 5(2), 197-208.

Úbeda-Colomer, J., Molina, P., y Campos, J. (2016). Las actividades físico-deportivas en la Universitat de València: Percepción del alumnado con discapacidad. Actividad Física y Deporte: Ciencia y Profesión, 25, 59-72.

Vizoso, C.M., y Arias, O. (2016). Estresores académicos percibidos por estudiantes universitarios y su relación con el burnout y el rendimiento académicos. Anuario de Psicología, 46(2), 90-97.

Zumeta, L.N., Basade, N., Telletxea, S., Amutio, A., y Bobowik, M. (2016). Flujo compartido en actividades colectivas físico-deportivas y bienestar subjetivo. European Journal of Investigation in Health, Psychology and Education, 6(3), 151-166.

Sebire SJ, Standage M, Vansteenkiste M. (2008). Development and validation of the Goal Content for Exercise Questionnaire. J Sport Exerc Psychol. 2008; 30: 353-377.

Maslach C, Jackson SE. Maslach Burnout Inventory Manual. 2 a ed. Palo Alto, California: Consulting Psychologists Press; 1986.

Schaufeli WB, Martínez IM, Pinto AM, Salanova M, Bakker AB. Burnout and engagement in university students. A Cross National Study. J Cross Cult Psychol. 2002; 33(5): 464481.

Recibido: 8 de abril de 2018

Recepción Modificaciones: 11 de mayo de 2018

Aceptado: 14 de mayo de 2018 